\title{
MOTIVACIÓN Y SATISFACCIÓN LABORAL EN CENTROS DE ENSEÑANZA SEGÚN EL PUESTO DE TRABAJO
}

\section{MOTIVATION AND LABOR SATISFACTION IN EDUCATION'S CENTERS ACCORDING TO THE WORKING PLACE}

\author{
Ma Inmaculada Fernández Ávalos, Laura López Rivas, Elena Martín Barranco, Marina \\ Ortiz López, Irene Pacheco Yepes y María Rodríguez Bocanegra. Universidad de \\ Granada \\ Artículo dirigido por: Inmaculada Valor Segura. Departamento de Psicología Social. Universidad de Granada \\ Fecha de recepción: 19 de marzo de 2014 \\ Fecha de revisión: 19 de marzo de 2014. \\ Fecha de aceptación: 11 de abril de 2014.
}

\section{RESUMEN}

El objetivo de esta investigación es mostrar el impacto que determina un puesto de trabajo concreto en la motivación de incentivos y la satisfacción laboral en centros de enseñanza de Granada. Por un lado, la motivación laboral se entiende como un proceso que activa, orienta, dinamiza y mantiene la conducta de los trabajadores para conseguir una meta. Por otro lado, la satisfacción laboral se entiende como la actitud que mantiene el individuo hacia su trabajo. De acuerdo con esto, se intentará establecer qué puesto de trabajo dentro de la jerarquía implica una mayor motivación y satisfacción laboral.

Palabras clave: Motivación laboral, satisfacción laboral, puesto de trabajo.

\begin{abstract}
The objective of this research is to show the impact that determines a concrete working place in the incentives motivation and the labor satisfaction in education's centers in Granada. On the one hand, the labor motivation is understood as a process that activates, orientates, stirs into action and supports the conduct of workers to obtain a goal. On the other hand, the labor satisfaction is understood as the attitude that the individual supports towards his work. In agreement with this, one will try to establish what working place inside the hierarchy implies a major motivation and labor satisfaction.
\end{abstract}

Keywords: labor motivation, labor satisfaction, working place.

\section{INTRODUCCIÓN}

Los psicólogos sociales se han interesado mucho a lo largo de la historia por la motivación. Los seres humanos necesitan de la motivación para realizar todas las actividades del día a día. En las organizaciones, la motivación de los trabajadores que la conforman es fundamental para obtener beneficios y buenos resultados. Se puede decir que la motivación laboral hace referencia a la fuerza psicológica que impulsa a las personas a iniciar, mantener y mejorar sus tareas laborales. Por ello, en el ámbito laboral es importante conocer las causas que estimulan la acción humana, ya que 
mediante el manejo de la motivación, entre otros aspectos, los administradores pueden operar estos elementos a fin de que su organización funcione adecuadamente y los miembros se sientan más satisfechos (López Mas, 2005).

Resulta realmente difícil encontrar un único criterio para establecer una clasificación coherente de la enorme cantidad de modelos y teorías de la motivación laboral existentes. Posiblemente, el criterio más adecuado por la gran cantidad de ventajas didácticas que ofrece y un reducido número de problemas taxonómicos ocasionados, sea la progresión cronológica que diferencia entre teorías de contenido, centradas en qué es lo que motiva en el ámbito laboral al individuo, y teorías de procesos, que muestran interés en cómo se motivan los trabajadores. Por un lado, entre las teorías de contenido se puede encontrar la Teoría de la jerarquía de necesidades de Maslow (1954), que defiende que el comportamiento humano se encuentra motivado por cinco grupos de necesidades, ordenados jerárquicamente. Otra de ellas es la Teoría de las necesidades sociales de McClelland (1961), la cual se centra en las necesidades resultantes de las relaciones sociales. Asimismo, la Teoría de los dos factores de Herzberg (1959) diferencia entre dos tipos de factores, los motivadores cuya presencia produce satisfacción y los higiénicos, que tienden a reducir la insatisfacción. También destacan las Teorías de necesidades y control, que considera la realización de los individuos depende de la consecución de la regulación de su comportamiento laboral en función de motivadores internos, siendo superfluo el control externo. Por último, referente a las teorías de contenido, se destaca el Modelo de las características del trabajo de Hackman y Oldham (1976), que afirma que determinadas características laborales generan estados psicológicos que repercuten en la motivación, satisfacción y eficacia. Por otro lado, entre las teorías de procesos se puede encontrar la Teoría camino-meta de Georgopoulos, Mahoney y Jones (1957) que considera que el rendimiento laboral está condicionado por la motivación, influenciada a su vez por los objetivos personales establecidos por cada trabajador. Con respecto a la Teoría de la expectativa-valencia (1964) para Vroom, la fuerza o motivación (F) depende tanto de la expectativa de conseguir los resultados (E) como de la valencia concedida a los mismos (V). Finalmente, cabe destacar la Teoría del establecimiento de metas de Locke y Bryan (1968) que establece que la motivación, y como consecuencia el nivel de ejecución, depende de los objetivos racionales y conscientes que los individuos se proponen alcanzar.

Existen numerosos factores que correlacionan significativamente con la motivación laboral como la satisfacción laboral, el estilo de liderazgo y el clima de comunicación (Cuadra-Peralta y Veloso-Besio, 2010), el cual es un factor esencial que determina tanto la motivación como la satisfacción de los empleados, tal y como lo han demostrado determinados estudios (Daly y Koprinek, 1982; Hernández, 2008).

La motivación, según la escala de motivaciones psicosociales (MPS) tiene 5 componentes básicos: el nivel de activación y necesidad, el nivel de expectativa, el nivel de ejecución, el nivel de satisfacción y, por último, el valor del incentivo de cada sujeto, factor en el que nos centraremos en comentar en esta investigación (Fernandez-Seara, 1996).

Según Barnat (1995), compensar es "igualar en sentido opuesto el efecto de una cosa con el de otra" o "dar alguna cosa o hacer beneficio en resarcimiento de algún daño causado". En ambas definiciones se puede encontrar un elemento central, la idea de intercambio y retribución (Bedodo y Giglio, 2006).

El concepto de compensación incluye todo tipo de recompensas, tanto extrínsecas como intrínsecas, que consideran elementos monetarios y no monetarios, y son 
recibidas por el empleado como resultado de su trabajo en la organización. Las recompensas extrínsecas se refieren al sueldo base o salario, incentivos o bonos y beneficios distribuidos directamente por la organización. Las recompensas intrínsecas se refieren a recompensas internas del individuo, derivadas de su involucración en ciertas actividades o tareas, como son: satisfacción laboral, compromiso, autonomía, oportunidades de crecimiento y aprendizaje (Villanueva y González, 2005).

Por otro lado, un factor determinante en la vida laboral de las personas y su rendimiento es la satisfacción laboral. Según Lawler III (1971), la satisfacción laboral está influenciada por cómo de satisfechos están los empleados con los tipos de recompensas que reciben por sus trabajos. Esto pone de manifiesto que las recompensas intrínsecas y extrínsecas, conjuntamente, inciden en la motivación, ya que satisfacen necesidades distintas. Esto significa, por ejemplo, que el dinero no compensará un trabajo aburrido y repetitivo, así como un trabajo interesante no compensará un sueldo bajo (Bedodo y Giglio, 2006).

En una investigación realizada en 1987 se midió la Satisfacción laboral utilizando el Cuestionario General de Satisfacción en Organizaciones Laborales (S-V) con 82 ítems, (Meliá, Zornoza, Sanz, Morte y González, 1987). De su factorización se obtuvieron seis factores referentes a satisfacción:

1. Satisfacción con la supervisión y la participación en la organización.

2. Satisfacción con el ambiente físico del lugar de trabajo.

3. Satisfacción con las prestaciones materiales y recompensas complementarias.

4. Satisfacción intrínseca del trabajo.

5. Satisfacción con la remuneración, las prestaciones básicas y la seguridad de empleo.

6. Satisfacción con las relaciones interpersonales en el trabajo, comprende las relaciones con las personas del mismo nivel jerárquico, así como con los subordinados y superiores.

Todas estas satisfacciones están asociadas a la posición ocupada dentro de la jerarquía. Asimismo, personas con un punto medio o alto en la jerarquía organizacional tendrán una mayor satisfacción laboral, puesto que reciben un salario mayor, disponen de un mayor nivel de autonomía, desempeñan tareas más creativas y complejas, etc., que personas pertenecientes a un nivel inferior en esta jerarquía (Meliá et al, 1987).

En el presente estudio se intenta establecer una relación entre motivación, principalmente asociada a incentivos, y satisfacción laboral en los centros de enseñanza dependiendo de la posición jerárquica ocupada por cada trabajador.

Por lo tanto nuestras hipótesis serían:

- Los cargos directivos tendrían mayor motivación y satisfacción laboral que el resto de los cargos.

- Los profesores interinos tendrán una mayor motivación que los profesores convencionales pero no así en la satisfacción.

- Los cargos más inferiores en la jerarquía (conserjes, limpiadores, etc.) tendrán menos motivación y satisfacción laboral.

- Una jornada laboral completa será más satisfactoria que una jornada a tiempo parcial.

- La antigüedad en un mismo centro de enseñanza produce un mayor nivel de satisfacción y motivación. 


\section{MÉTODO}

Participantes

La muestra estuvo compuesta por trabajadores pertenecientes a centros educativos con niveles jerárquicos diferentes con un total de 105 sujetos (46 hombres y 59 mujeres). Dentro de la cual había 24 directores y jefes de estudios que corresponde al nivel más alto; 42 profesores que constituyen el siguiente nivel, seguido de 19 profesores interinos y el último escalón lo conformarían 19 conserjes, limpiadoras, etc. Todos ellos con edades comprendidas entre 22 y 65 años, siendo la edad media de 43,04 y una desviación típica de 10,54. Se trataba de trabajadores procedentes de doce centros públicos de enseñanza, cinco de Enseñanza Primaria Obligatoria y siete de Enseñanza Secundaria Obligatoria, de la provincia de Granada.

\section{Diseño}

El presente estudio utiliza un diseño correlacional en el que se pretende realizar una asociaciónentre un puesto de trabajo concreto y su nivel de motivación laboral, y por otro lado, mostrar la relación existente entre dicho puesto de trabajo y su nivel de satisfacción laboral.

\section{Instrumentos}

En la recogida de datos se utilizaron dos instrumentos de medida:

Por un lado, se utilizó la dimensión que engloba el valor del incentivo para cada sujeto de la Escala de Motivaciones Psicosociales (MPS) (Fernández-Seara, 2009). Esta dimensión está compuesta por un total de 21 ítems, que recogen 21 incentivos diferentes y se responden con una escala Likert de 2 (normal) a 4 (muchísimo). La escala muestra un valor para el coeficiente alfa de Cronbach de 0.80 .

Por otro lado, se empleó la versión más sintética (S10/12) del Cuestionario de Satisfacción Laboral (Meliá et al., 1986), para medir el grado de satisfacción presente en función de las diversas condiciones laborales. Este cuestionario está compuesto por un total de 12 ítems que se responden con una escala Likert que oscila entre 1 (muy insatisfecho) y 7 (muy satisfecho). La escala muestra un valor para el coeficiente alfa de Cronbach de 0.80 . Este cuestionario, a su vez, engloba un apartado para la recogida de datos descriptivos (ocupación, categoría laboral, sexo, edad, nivel de estudios, situación laboral, horario, nivel jerárquico y antigüedad).

\section{Procedimiento}

La recogida de datos se llevó a cabo en los respectivos centros de enseñanza de los participantes en su correspondiente horario de trabajo. En primer lugar, se hizo énfasis en que la participación en el estudio sería de manera anónima y confidencial y además se les dieron las instrucciones oportunas para poder realizar las diferentes pruebas proporcionadas. El siguiente paso una vez confirmada su participación en la investigación fue proporcionarles los distintos instrumentos de evaluación utilizados en este estudio, anteriormente comentados, cuya duración no sobrepasó los 20-25 minutos. Finalmente, una vez acabada la administración y realización de dichas pruebas se les agradeció su colaboración en el estudio. 


\section{RESULTADOS}

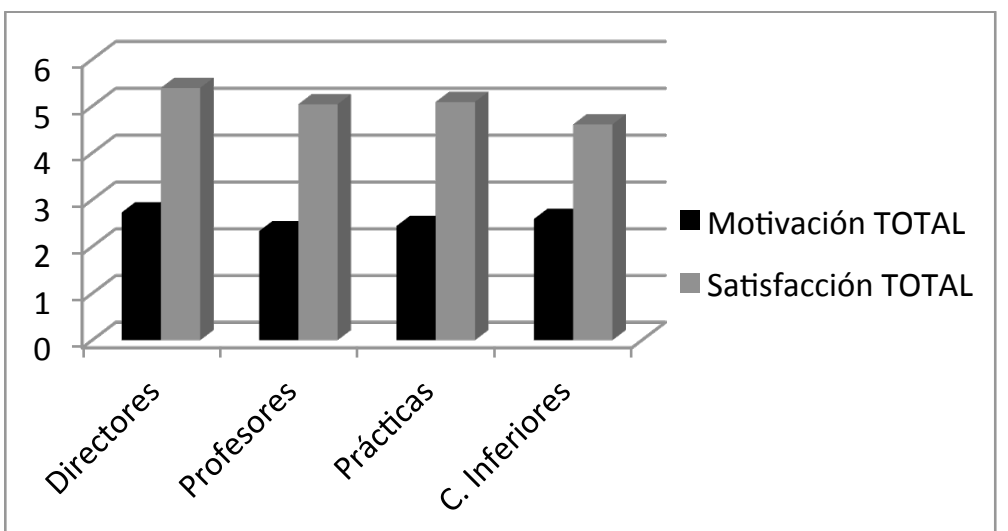

Gráfica 1. Comparación en los niveles de Motivación y Satisfacción en los diferentes niveles de la jerarquía.

Para llevar a cabo este análisis de resultados se han realizado las medias y desviaciones típicas de todos los niveles de la jerarquía y las correlaciones existentes entre motivación y satisfacción de cada nivel, utilizando la correlación de Pearson, teniendoen cuenta que $(p<.05)$.

En primer lugar de altos cargos se han obtenido las siguientes medias y desviaciones típicas: en motivación $(M=2,73 ; S D=0,53)$; y en satisfacción $(M=5,39 ; S D=0,61)$. En cuanto a los profesores, se ha obtenido en motivación $(M=2,35 ; S D=0,80)$ y en satisfacción $(M=5,04 ; S D=0,93)$. Por otro lado los profesores interinos tienen en motivación $(M=2,44 ; S D=0,68)$ en satisfacción $(M=5,09 ; S D=0,95)$ y por último los cargo inferiores han obtenido en motivación $(M=2,59 ; S D=0,61)$ y en satisfacción $(M=$ $4,61 ; \mathrm{SD}=1,50)$.

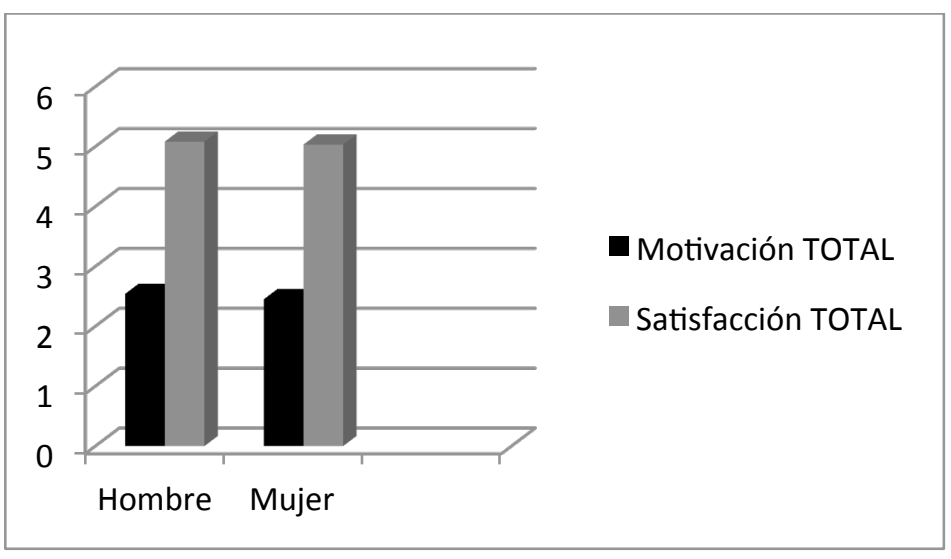

Gráfica 2.Comparación de los niveles de Motivación y Satisfacción dependiendo del sexo.

En este caso se ha llevado a cabo el análisis anterior, obteniendo como resultado en hombres en la variable motivación $(M=2,54 ; S D=0,61)$ y en la variable satisfacción $(M=5,1 ; S D=0,92)$. Por otro lado, las mujeres han obtenido en motivación $(M=2,45$; $S D=0,76)$ y en satisfacción $(M=5,03 ; S D=1,09)$. 
Tabla 1. Análisis correlacional de las variables

\begin{tabular}{lll}
\hline & MOT & ST \\
\hline 1.HT & .099 & $.254^{* *}$ \\
2.MT & .068 & .148 \\
3.ED & -.118 & .156 \\
\hline
\end{tabular}

Nota: Mot: Motivación; ST: Satisfacción; HT: Horas trabajadas; MT: Meses Trabajados; ED: Edad. $* p<0.05 * * p<0.01$

En el análisis de correlación (Tabla 1) se puede observar que las variables horas trabajadas, meses trabajados y la edad no mantienen correlaciones significativas ni con la variable motivación laboral, ni satisfacción laboral. A excepción de la correlación de horas trabajadas con satisfacción laboral, ya que hemos obtenido que la satisfacción laboral correlaciona con las horas trabajadas muy significativamente y de manera positiva $(r=-.254 ; p<0,01)$. Hay que tener en cuenta que la correlación entre la edad y la motivación es negativa aunque no sea significativa.

\section{DISCUSIÓN}

Nuestra primera hipótesis consistía en comprobar la existencia de un mayor grado de motivación y satisfacción laboral en los altos cargos. No hemos obtenido diferencias significativas respecto al resto de los grupos, aunque se puede observar como la satisfacción y la motivación son mayores que el resto. Esto puede deberse a la obtención de un mayor número de incentivos como la retribución de un salario más elevado, mejores condiciones laborales, mayor estabilidad y poder, etc. tal como defiende Meliá et al. (1987) en su investigación.

En nuestra segunda hipótesis se ha demostrado que los profesores interinos tienen una motivación mayor que el resto de profesores convencionales, aunque el resultado no ha sido significativo. En cambio se ha encontrado mayores niveles de satisfacción en profesores interinos. Puede deberse a la incorporación temprana en un puesto de trabajo deseado. Como indican Meliá et al. (1987), en las jerarquías influye la satisfacción intrínseca del trabajo, que se relaciona con la posibilidad de crear o de desempeñar un buen trabajo ya que la persona se considera que destaca en ese ámbito. Es lo que le sucedería a los profesores interinos, ya que el trabajo le produce satisfacción en sí mismo.

Otra de nuestras hipótesis expone que los cargos que se encuentran en la base de la jerarquía (conserjes, limpiadores) tendrán menor grado de motivación y de satisfacción laboral. A pesar de no haber encontrado diferencias significativas, en el caso de la motivación, se ha obtenido un mayor grado con respecto a los profesores convencionales e interinos, posiblemente debido al desempeño de un trabajo menos rutinario y más sociable en concordancia con la teoría que defiende McClelland (1961). En cuanto a la satisfacción, ésta si ha alcanzado un nivel más bajo en relación al resto de trabajadores, quizá por la ganancia de un salario más reducido y por unas condiciones laborales inferiores, entre otros factores tal y como afirman Meliá et al. (1987) en su teoría donde relaciona la satisfacción con las prestaciones materiales y recompensas complementarias.

Aludiendo a la hipótesis que hacía referencia a que una jornada laboral completa sería más satisfactoria que una a tiempo parcial, nuestros resultados muestran diferencias significativas al respecto, corroborando dicha hipótesis. La mayor satisfacción en profesionales con jornada completa se podría relacionar con una mayor cantidad de recompensas tanto extrínsecas como intrínsecas, como por ejemplo un salario 
elevado, un mayor grado de implicación y compromiso con la organización, además de ofrecer un número más elevado de oportunidades para crecer laboralmente. Esto pone de manifiesto el supuesto de Lawer III (1971) que señala que la presencia de ambos tipos de recompensas permite saciar distintos tipos de necesidades, y a su vez, fomenta el crecimiento de la satisfacción laboral.

Nuestra última hipótesis defendía que la antigüedad en un mismo centro de enseñanza produciría un mayor nivel de satisfacción y motivación lo cual ha sido apoyado por los datos que hemos recabado, aunque no muestran que estas diferencias sean significativas. Esto puede deberse a que el trabajador ya tiene confianza en el trabajo que realiza, por ello los resultados son mejores y está satisfecho de lo que obtiene. Además, su motivación aumenta cuando sus objetivos previstos eran alcanzados, sobre todo si eran de gran importancia para el trabajador, tal y como indica la Teoría de la expectativa-valencia (1964) y/o la Teoría de Locke y Bryan (1968).

De manera casual, hemos encontrado una pequeña diferencia en la cantidad de satisfacción y motivación entre ambos sexos, teniendo el género masculino mayor cantidad de ambas variables que el género femenino. En futuras investigaciones se podría tener esta variable en cuenta para averiguar si las diferencias son significativas e investigar las causas de tales diferencias.

Con respecto a la edad, podemos observar que los trabajadores más jóvenes tienen un mayor nivel de motivación pero un menor nivel de satisfacción. Una posible explicación es que al ser más jóvenes tienen más ganas de empezar a trabajar en aquello en lo que se han formado en los últimos años, así su motivación es mayor. Además, tienen un menor grado de satisfacción ya que al no tener tanta experiencia pueden verse sobrecargados en sus tareas y no saber cómo llevar algunas situaciones de la forma lo más eficazmente posible.

Por último, cabe destacar que la ausencia de corroboración de las hipótesis planteadas puede ser consecuencia de la utilización de una reducida y desequilibrada muestra, así como de una falta de aleatorización en la selección de la misma. Además de la incapacidad de poder controlar los sesgos que posiblemente hayan aparecido en el estudio como consecuencia de condiciones externas, como la presentación de los cuestionarios en periodo de evaluaciones, caracterizado por la disponibilidad de tiempo reducida.

\section{BIBLIOGRAFÍA}

Barnat, J. (1995). Diccionario enciclopédico Nauta Maior. Barcelona, España: Ediciones Nauta.

Bedodo Espinoza, V. y Giglio Gallardo, C. (2006). Motivación laboral y compensaciones: una investigación de orientación teórica. Tesis doctoral inédita. Facultad de Ciencias Sociales. Universidad de Chile.

Cuadra-Peralta, A.A. y Veloso-Besio, C.B. (2010). Grado de supervisión como variable moderadora entre liderazgo y satisfacción, motivación y clima organizacional. Ingeniare. Revista chilena de ingeniería, 18(1), 15-25. 
Daly, John A. y Koprinek, J. (1982). Organizacional Communications: A Review Via Operationalizations. En Greenbaum, Howard H. y Falcione, Raymond L. (Eds.). Organizational Communication: Abstracts, Analysis, and Overview. Beverly Hills: Sage Publications.

Fernández-Seara, J.L.(1996). MPS escala de motivaciones psicosociales. Madrid: TEA.

Georgopoulos, B.S., Mahoney, G.M. y Jones, N.W.Jr. (1957). A path-goal approach to productivity. Journal of Applied Psychology, 41(6), 345-353.

Hackman, J.R. y Oldham, G.R. (1976). Motivation through the Design of Work: Test of a Theory. Organitazional Behavior and Human Performance, 16, 250-279.

Hernández, D. (2008). La relación entre el sistema formal de comunicación interna, el clima de comunicación y la identificación de los empleados en las organizaciones. Tesis doctoral inédita. Universidad de Barcelona. España

Herzberg, F., Mausner, B. y Snyderman, B.B.(1959). The motivation to work. New York, NY: John Wiley \& Sons.

Lawler, E. E. (1971). Pay and organizational effectiveness: A psychological view. New York: McGraw-Hill.

Locke, E. A. y Bryan, J. (1968).Goal setting as a determinant of the effects of knowledge of score in performance. American Journal of Psychology, 81, 398406.

López Mas, J. (2005).Motivación laboral y gestión de recursos humanos en la teoría de Frederick Herzberg. Gestión en el Tercer Milenio, 8(15),25-36.

Maslow, A. H. (1954). Motivation and Personality. New York: Harper and Row.

McClelland, D. C.(1961).The achieving society. New York: The Free Press.

Meliá, J.L., Peiró, J.M., y Calatayud, C. (1986). Cuestionario General de Satisfacción en Organizaciones Laborales: Estudios factoriales, fiabilidad y validez. Millars. Rev. de Filosofía, Psicologia y Ciencias de la Educación, 11(3-4), 43-78.

Meliá, J.L., Zornoza, A., Sanz, M.J., Morte, M.P., y González, V. (1987). La incidencia de los factores del conflicto de rol y de la ambigüedad de rol sobre los factores de la satisfacción laboral. Actas del Segundo Congreso Nacional de Evaluación Psicológica. Madrid. 287.

Villanueva, A. y González, E. (2005). Gestión en las Compensaciones I. Manual del participante. Santiago, Chile.

Vroom, V.H. (1964).Work and motivation.New York: Wiley. 